NASA Technical Memorandum 86374

\title{
VISCOUS EFFECTS ON TRANSONIC AIRFOIL STABILITY AND RESPONSE
}

H. M. BERRY

J. T, BATINA

$T, Y, Y A N G$

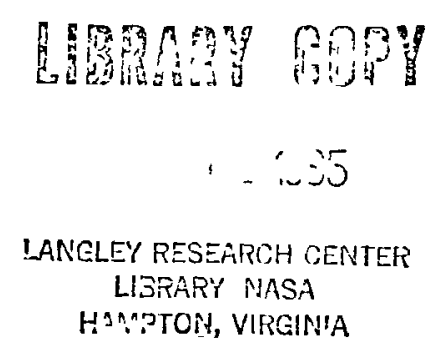




\author{
H. M. Berry \\ Purdue University \\ West Lafayette, Indiana \\ J. T. Batina \\ NASA Langley Research Center \\ Hampton, Virginia
}

T. Y. Yang

Purdue University

West Lafayette, Indiana

\section{Abstract}

Viscous effects on transonic airfoil stability and response are investigated using an integral boundary layer model coupled to the inviscid XTRAN2L transonic small-disturbance code. Unsteady transonic airloads required for stability analyses are computed using a pulse transfer-function analysis including viscous effects. The pulse analysis provides unsteady aerodynamic forces for a wide range of reduced frequency in a single flowfield computation. Nonlinear time-marching aeroelastic solutions are presented which show the effects of viscosity on airfoil response behavior and flutter. Effects of amplitude on time-marching responses are demonstrated. A state-space aeroelastic model employing Pade approximants to describe the unsteady airloads is used to study the effects of viscosity on transonic airfoil stability. State-space dynamic pressure rootloci are in good overall agreenent with timemarching damping and frequency estimates. Parallel sets of results with and without viscous effects reveal the effects of viscosity on transonic unsteady airloads and aeroelastic characteristics of airfoils.

\section{Nomenclature}

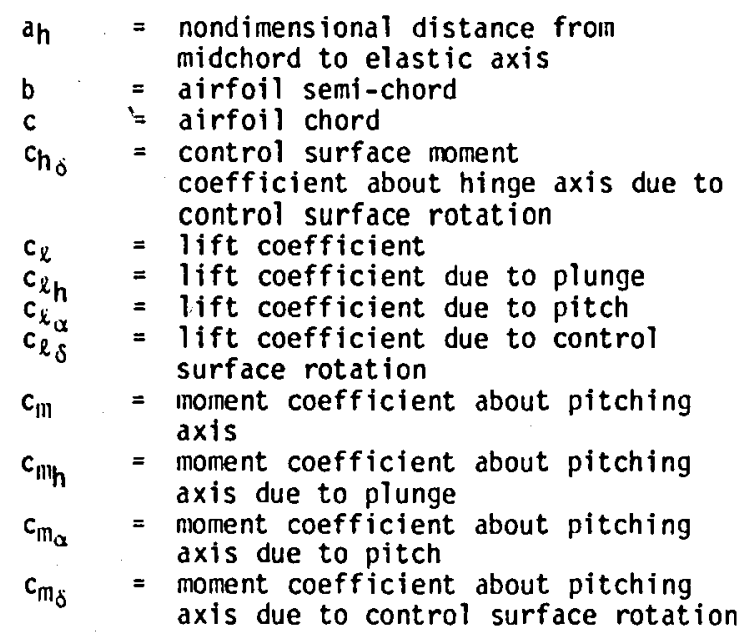

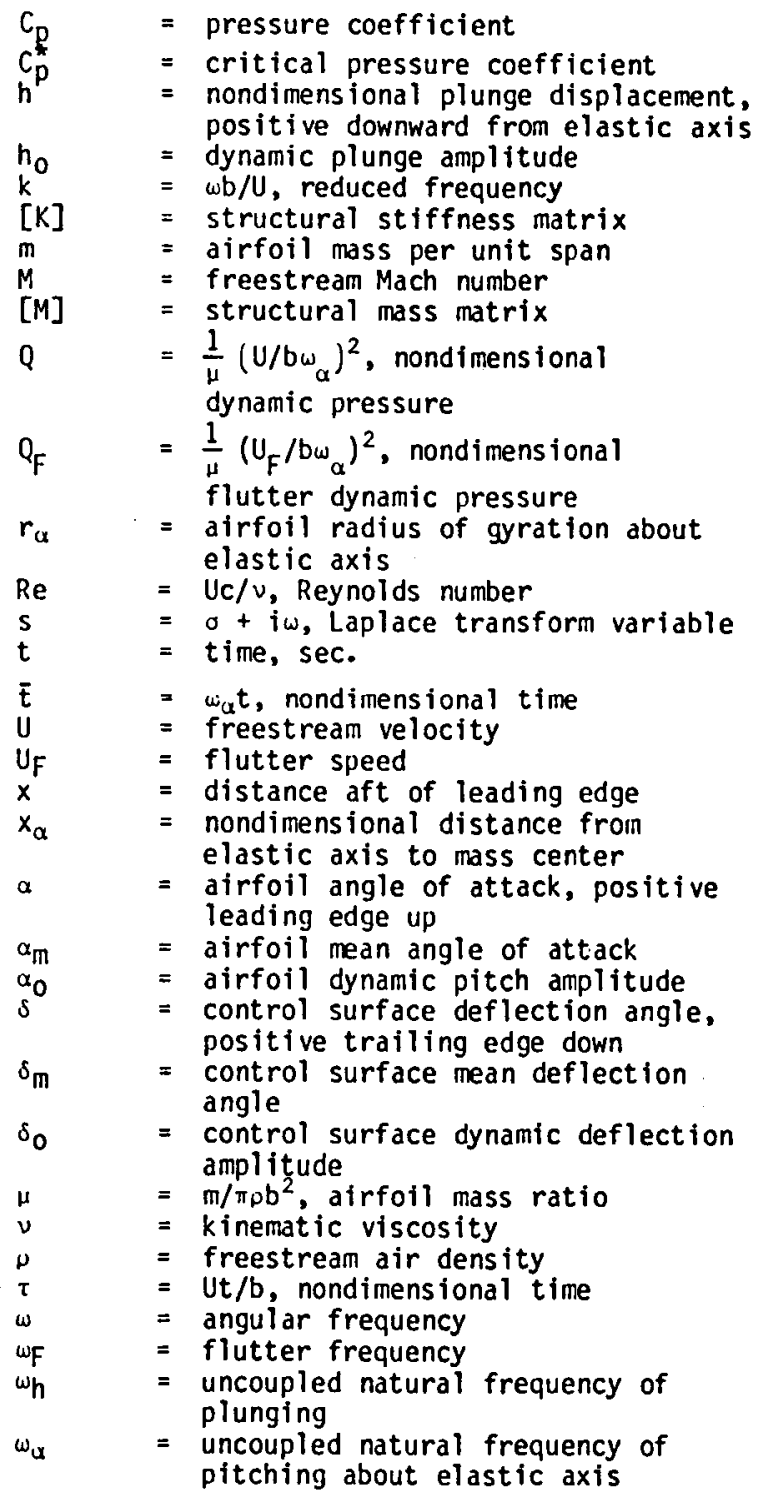

\section{Introduction}

Research directed at better understanding of aerodynamic and aeroelastic phenomena at transonic speeds has increased greatly in recent years. These developments have been made possible by the advances made in computer power and numerical solution techniques. 1 Much effort 
has been put into the development of accurate yet cost efficient methods of calculating unsteady transonic afrloads as well as the prediction of aeroelastic characteristics such as flutter and divergence.

Solutions for inviscid unsteady transonic flowfields about oscillating airfoils have been made possible using computer codes such as LTRAN2. 2 While the inviscid flowfields predicted by LTRAN2 give valid results for many cases, these solutions are not adequate when viscous effects are important. Rizzetta ${ }^{3}$ incorporated a viscous boundary layer model into the LTRAN2 code using a viscous ramp model and Green's lagentrainment equations, an integral boundary layer model. A non-iterative implicit technique was used to couple the quasi-steady viscous equations with the inviscid potential flow equations. Guruswamy and Goorjian ${ }^{4}$ applied this modified version of LTRAN2 to investigate the effects of viscosity on transonic aerodynamic and aeroelastic characteristics of oscillating airfoils. Two degree-of-freedom (plunge and pitch) typical section flutter speeds were presented for the conventional NACA 64A010 Ames airfoil (herein referred to as NACA 64A010A) and the supercritical $M B B-A 3$ alrfoil. As many as 8000 time steps per cycle of oscillatory motion were required in the viscous lag-entrainment calculations to obtain reasonably converged results. Steady and unsteady viscous results agreed better with experiment than did comparable inviscid solutions.

Houwink ${ }^{5}$ developed a procedure for an explicit coupling of the unsteady transonic flow and turbulent boundary layer computations, and incorporated them into the LTRAN2-NLR ${ }^{6}$ code. Satisfactorily converged results were obtained in as few as 120 time steps per cycle using the resulting LTRANV ${ }^{5}$ code. More recently, Howlett has modified the viscous coupling procedure of Rizzetta for application to the XTRAN2L ${ }^{8}$ general frequency transonic small-disturbance code. An integration of Green's lag-entrainment equations is performed from a specified transition point on the airfoil to the downstream boundary. The viscous ramp model is discarded and boundary layer smoothing is used to reduce flow instabilities. Iteration of an explicitly coupled viscous boundary layer solution with the inviscid outer flow at each time step provides converged flowfield solutions in as few as one or two iterations. In general, converged oscillatory solutions including viscous effects may be obtalned in three cycles of motion with 360 time steps per cycle. Viscous solutions are obtained in computer times approximately twice that of the comparable inviscid solution. Therefore, a more practical and affordable assessment of the effects of viscosity on transonic unsteady aerodynamic forces and aeroelastic characteristics of oscillating airfolls is now possible. The explicitly coupled viscous-inviscid procedure of Ref. 7 is used in the present study to calculate time-marching flutter solutions and transonic unsteady airloads required for aeroelastic stability analyses.

In the present study, airfoil stability analyses are performed using state-space aeroelastic modeling such as that reported in Refs. 9-12. Edwards, et al. ${ }^{9}$ used a state-space model employing Pade approximants to model the unsteady airloads and demonstrated good agreement with a time-marching technique for a demonstrated that such locally linear procedures may be used with airloads derived from a transonic small-disturbance code. Batina and Yang 11,12 used a similar procedure to study the transonic aeroelastic stability and response behavior of airfoils with active controls.

The purpose of this paper is to further investigate the effects of viscosity on transonic airfoll stability and response. The object ives of the study were: (1) to investigate application of the pulse transfer-function analysis of Seidel, Bennett, and Whitlow ${ }^{13}$ to treat airloads including viscous effects; (2) to investigate application of the nonlinear timemarching flutter solution procedure of Edwards, et al. 9 to transonic airfoil response including viscous effects; (3). to determine the effects of amplitude on inviscid and viscous time-marching flutter solutions; (4) to assess the accuracy of state-space aeroelastic modeling to predict airfoil stability including viscous effects by comparison with time-marching analyses; and (5) to apply state-space modeling to study the effects of viscosity on transonic airfoil stability. Resuits are presented for the NACA 64A010A, MBB-A3, and NACA $64 A 006$ airfoils. Time-marching response and state-space stability analyses are performed for the NACA 64AO1OA and MBB-A3 airfoils. Parallel sets of results are presented with and without viscous effects to determine the effects of viscosity on transonic airfoil stability and response.

\section{Computational Procedures}

\section{XTRAN2L Transonic Code}

The finite-difference code XTRAN2L solves the transonic small-disturbance potential equation and thus provides predictions of unsteady transonic flowfields about oscillating airfoils. The inviscid algorithm, however, tends to overpredict the shock strength and locates the shock too far aft for cases where viscous effects are important. In order to predict both shock strength and location more accurately, viscous corrections are needed. The effects of viscosity are accounted for by an integration of Green's lag-entrainment equations along the airfoil from a transition point selected at $10 \%$ chord to the downstream boundary. Iteration of the viscous-inviscid solution may be performed, ensuring converged flowfield results at each time step.

\section{Pulse Transfer-Function Analysis}

Unsteady aerodynamic forces required for stability calculations are computed using the pulse transfer-function analys is available in XTRAN2L. This analysis is based on the assumption that the unsteady forces are locally linear about the nonlinear transonic mean flow. In the pulse analysis, the airfotl is given a small prescribed pulse in a given mode of motion and the aerodynamic transients are calculated. For pitch motion the pulse is given by 


$$
\alpha=\alpha_{m}+\alpha_{0} e^{-(\tau-17.5 \Delta \tau)^{2}}
$$

where $\Delta \tau$ is the nondimensional time step. Similar expressions describe the pulses for plunge and control surface motions. The unsteady aerodynamic transfer-function is then determined by dividing a fast Fourier transform (FFT) of the output force time history by the FFT of the input pulse motion. The pulse analysis provides unsteady aerodynamic forces for a wide range of reduced frequency in a single flowfield computation. This is in contrast to multiple flowfield computations required for calculating oscillatory forces for discrete frequencies. Both inviscid and viscous pulse transfer-function analyses are performed. A typical inviscid pulse analysis was computed with $\Delta \tau=5 \pi / 32$ and 1024 time steps while a typical viscous pulse analys is was computed with $\Delta \tau=2 \pi / 27$ and 2160 time steps.

To assess the accuracy of the pulse transfer-function analys is including viscous effects, unsteady aerodynamic forces were calculated for simple harmonic motion at several discrete values of reduced frequency $k$. Three cycles of oscillatory motion with 360 time steps per cycle and one viscous iteration per time step were used for most cases. For $k=0.05$, 720 time steps per cycle and two iterations per time step were required.

\section{Time-Marching Analys is}

The aeroelastic system considered consists of plunge and pitch degrees-of-freedom. The equations of motion may be written in matrix form as

$$
[M]\left\{\begin{array}{l}
\ddot{h} \\
\ddot{a}
\end{array}\right\}+[K]\left\{\begin{array}{l}
h \\
\alpha
\end{array}\right\}=\frac{Q}{\pi}\left\{\begin{array}{l}
-c_{\ell} \\
2 c_{m}
\end{array}\right\}
$$

where the dot denotes differentiation with respect to nondimensional time $\bar{t}$. Time-marching aeroelastic solutions of Eq. (2) are obtained both with and without viscous effects. In the time-marching analysis, the equations of motion are coupled with the aerodynamic solution procedure of XTRAN2L for simultaneous time-integration. Equation (2) is numerically integrated in time using the modified statetransition matrix integrator of Edwards, et al. 9 Details of the solution procedure may be found in Ref. 9. A typical inviscid analysis was performed with $\Delta t=0.0004$; a typical viscous solution was obtained with $\Delta t=0.0003$. In general, several aeroelastic transients were calculated for a range of nondimensional dynamic pressure Q. Values of $Q$ were selected which resulted in subcritical damped responses and supercritical diverging responses. The nondimensional flutter dynamic pressure, $Q_{F}$, was calculated by interpolation and then confirmed by obtaining neutrally stable responses.

Damping and frequency of the aeroelastic modes are estimated from the transient response curves using the method of Bennett and Desmarais. 14 These modal estimates are determined by a least squares curve-fit of the aero- elastic transients using complex exponential functions of the form

$$
\begin{aligned}
& x(\bar{t})=a_{0}+\sum_{j=1}^{m} e^{\left(\frac{\sigma}{\omega_{\alpha}}\right){ }_{j} \bar{t}}\left[a_{j} \cos \left(\frac{\omega}{\omega_{\alpha}}\right)_{j} \bar{t}\right. \\
& \left.+b_{j} \sin \left(\frac{\omega}{\omega_{\alpha}}\right)_{j} \bar{t}\right]
\end{aligned}
$$

Damping and frequency estimates from the timemarching analysis are plotted in the complex s-plane.

\section{Padé Model}

Aeroelastic stability analyses are performed using a state-space aeroelastic model, termed the Pade model, similar to that of Refs. 11 and 12. The locally 1 inear Pade model provides a relatively inexpensive determination of airfoil stabllity while retaining the nonlinear properties of the mean flow. Transonic unsteady airloads required by this analys is are determined by inviscid and viscous pulse analyses. The Pade model is derived by assuming linear superposition of airloads due to airfoil plunge and pitch motions. The required airloads are approximated by curve-fitting the XTRAN2L unsteady aerodynamic forces with a Pade approximating function. ${ }^{11}$ The function may then be rewritten as a set of ordinary differential equations, which when coupled to the equations of motion, Eq. (2), and Laplace transformed leads to a linear first-order matrix equation

$$
\frac{s}{w_{\alpha}}\{z\}=[A]\{z\}
$$

where $\{z\}$ contains the displacements, velocities, and augmented states, and [A] is a real matrix of constant elements. Equation (4) is solved using linear eigenvalue solution techniques for specified values of $Q$. The resulting eigenvalues are plotted in a dynamic pressure "root-locus" type format and are compared with time-marching damping and frequency estimates in the complex s-plane.

\section{Resuits and Discussion}

Calculations were performed for the NACA 64A010A, MBB-A3, and NACA 64A006 airfoils. Airfoll coordinates required for aerodynamic computations were taken from Ref. 15. Computational conditions were selected to match the experimental conditions reported in Refs. 16-18. Transonic steady pressure distributions and unsteady aerodynamic forces were studied for all three airfoils for the computational conditions listed in Table 1 . Time-marching response and Pade model stability analyses were performed for the NACA 64A010A and MBB-A3 airfoils.

Aeroelastic results are presented for two example sets of structural parameter values. Example 1 is Case $A$ of Isogai ${ }^{19}$ which has bending and torsion modes similar to those of a streamwise section near the tip of a sweptback 
Table 1 Airfolls and computational conditions for

transonic aerodynamic and aeroelastic analyses.

\begin{tabular}{|l|c|c|c|c|c|}
\hline Airfoil & $\begin{array}{c}\text { Computational } \\
\text { Condition }\end{array}$ & $M$ & $\alpha_{m}$ & $\delta_{m}$ & $\operatorname{Re}$ \\
\hline NACA 64A010A & 1 & 0.796 & $-0.21^{\circ}$ & & $12.56 \times 10^{6}$ \\
\hline MBB-A3 & 2 & 0.765 & $1.50^{\circ}$ & & $6.0 \times 10^{6}$ \\
\hline MBB-A3 & 3 & 0.7557 & $1.30^{\circ}$ & & \\
\hline NACA 64A006 & 4 & 0.85 & $0^{\circ}$ & $0^{\circ}$ & $2.41 \times 10^{6}$ \\
\hline
\end{tabular}

wing. Example 2 is the structural configuration used by Edwards, et al. 9 (also termed Example 2 in Ref. 9) which has bending and torsion frequencies that are close together. Values for the structural parameters for the two examples are listed in Table 2.

\section{Transonic Steady Aerodynamic Results}

Steady flowfields were computed for use as initial conditions for unsteady aerodynamic calculations. Steady pressure distributions for the lower surface of the NACA 64A010A airfoil are shown in Fig. I along with a plot of the airfoil contour. Both inviscid and viscous XTRAN2L pressure distributions are presented and compared with the experimental data of Davis. 16 Calculations were performed at the experimental conditions $^{16}$ of $M=0.796, \alpha_{m}=-0.21^{\circ}$, and $\operatorname{Re}=12.56 \times 10^{6}$ which are herein termed Computational Condition 1 , as listed in Table 1 . All three sets of results are in reasonable agreement. In the region of the shock, the viscous computations are in better agreement with the experimental data than the inviscid computations. The calculated viscous shock is slightly weaker in strength and is located slightly upstream from the inviscid shock location. Viscous effects are relatively mild for this case.

Steady pressure distributions for the MBB-A3 airfoil are shown in Fig. 2 along with a plot of the airfoil contour. Computational results are obtained at two different sets of conditions for comparison with the experimental

Table 2 Structural parameter values for aeroelastic analyses

\begin{tabular}{|c|c|c|}
\hline \multirow{2}{*}{$\begin{array}{c}\text { Structural } \\
\text { Parameter }\end{array}$} & \multicolumn{2}{|c|}{ Example } \\
\cline { 2 - 3 } & 1 & 2 \\
\hline$a_{h}$ & -2.0 & -0.042 \\
$x_{\alpha}$ & 1.8 & -0.036 \\
$r_{\alpha}$ & 1.865 & 1.368 \\
$\mu$ & 60.0 & 60.0 \\
$\omega_{h}$ & 100.0 & 23.5 \\
$\omega_{\alpha}$ & 100.0 & 35.0 \\
\hline
\end{tabular}

data of Bucciantini, et al. 17 The first set corresponds to the uncorrected tunnel conditions 17 of $M=0.765, a_{n}=1.5^{\circ}$, and $\operatorname{Re}$ $=6.0 \times 10^{6}$ which are herein termed Computational Condition 2, as listed in Table 1. The second set corresponds to the design condition 17 of $M=0.7557$ and $\alpha_{m}=1.3^{\circ}$ which is herein termed Computational Condition 3 , as listed in Table 1. Computational Condition 3 allows inviscid computations to match the experimental pressure data and hence viscous calculations are not presented at this condition. Both inviscid and viscous solutions are presented at Computational Condition 2. As shown in Fig. 2, all four sets of results agree well along the lower surface of the airfoll except near the leading edge. For the upper surface, the XTRAN2L pressure distributions at computational Condition 2 indicate an overprediction of the shock strength and location, although the viscous computation is in slightly better agreement with experimental data than the inviscid computation. Differences between the viscous results and experiment may be attributed to the fact that the boundary layer model does not account for strong interaction between the shock wave and the boundary layer. Invisicid calculations performed at Computational Condition 3 , however, show much better agreement with experiment than either of the calculations performed at computational Condition 2 .

Similar comparisons of steady pressure distributions for the MBB-A3 airfoil at Computational Condition 3 have been reported by Guruswamy and Goorjian. ${ }^{4}$ Similar to the inviscid XTRAN2L results at Computational Condition 3 shown in $\mathrm{Fig}$. 2, the viscous LTRAN2 results of Ref. 4 are in good agreement with the experimental data of Ref. 17. Differences between XTRAN2L and LTRAN2 steady pressure distributions are attributed to the different grids and transonic scaling used in the two codes.

Steady pressure distributions for the lower surface of the NACA $64 A 006$ airfoil are shown in Fig. 3 along with a plot of the airfoil contour. Both inviscid and viscous XTRAN2L pressure distributions are presented for comparison with the steady experimental data of Zwaan.18 Computations were performed at the experimental conditions $^{18}$ of $M=0.85, \alpha_{m}=0^{\circ}, \delta_{m}=0^{\circ}$, and $\mathrm{Re}=2.41 \times 10^{6}$, which are herein termed Computational Condition 4 , as listed in Table 1. All three sets of results agree well except in the region of the shock. Here, the viscous computations are in better agreement with 


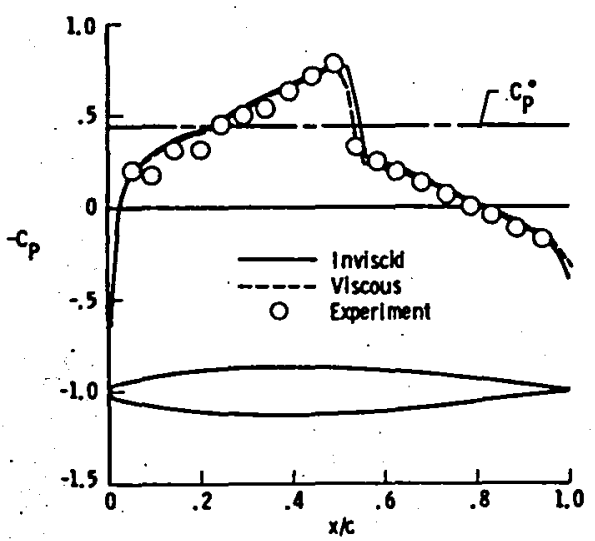

Fig. 1 Steady pressure distributions for the lower surface of the NACA 64A010A airfotl at $M=0.796$ and $a_{m}=-0.21^{\circ}$.

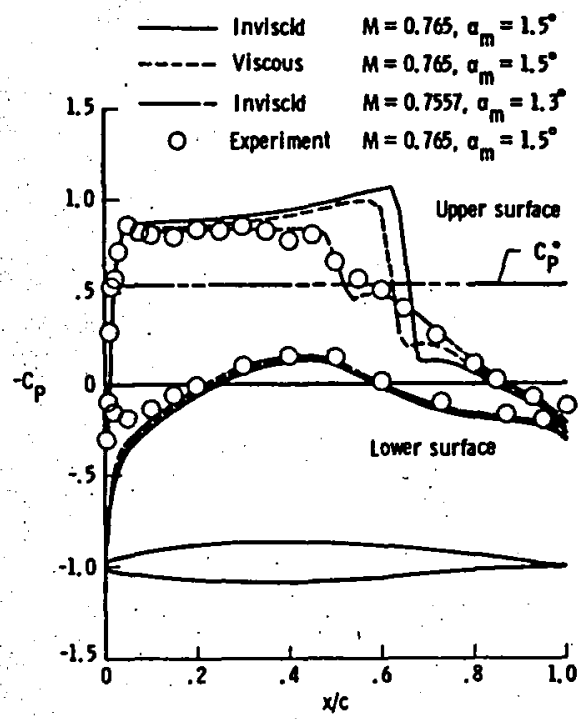

Fig. 2 Steady pressure distributions for the MBB-A3 airfoil at $M=0.765, a_{m}=1.5^{\circ}$ and $M=0.7557, \alpha_{m}=1.3^{\circ}$.

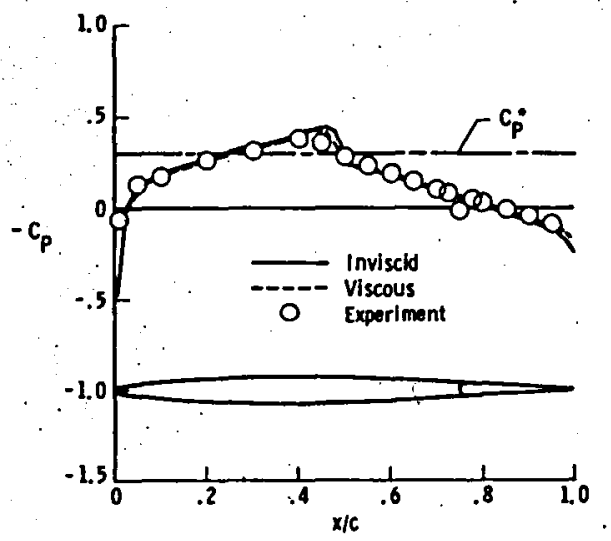

Fig. 3 Steady pressure distributions for the lower surface of the NACA 64A006 airfoll at $M=0.85, \alpha_{m}=0^{\circ}$ and $\delta_{m}=0^{\circ}$. experimental pressures than the inviscid computations. In the viscous solution, the shock is weaker and is located slightly upstream from the inviscid calculation. Viscous effects are relatively mild for this case.

\section{Transonic Unsteady Aerodynamic Results}

Unsteady aerodynamic forces required for Pade model stability calculations were computed using the pulse transfer-function analysis. Representative results for the 1 ift coefficient due to pitch, $c_{\ell}$, are plotted in Fig. 4 as a function of reduced frequency $k$. These results were obtained using the pulse analysis with viscous effects for the NACA 64A010A airfoil at Computational Condition 1 and a small pulse amplitude of $\alpha_{0}=0.1^{\circ}$. To assess the accuracy of the pulse analysis, oscillatory calculations including the effects of viscosity were performed for comparison. Results were obtained using an amplitude of $\alpha_{0}=0.1^{\circ}$ at eight values of reduced frequency $k=0.05,0.1$, $0.2,0.4,0.8,1.2,1.6$, and 2.0. As shown in Fig. 4, the pulse transfer-function is in excellent agreement with the oscillatory airloads: The excellent agreement between the two sets of results for this representative case clearly demonstrates the applicability of the pulse transfer-function analysis to include viscous effects.

To investigate the effects of viscosity on transonic unsteady airloads, inviscid and viscous pulse analyses are performed. A representative comparison between pulse results for $c_{l_{2}}$ is given in Fig. 5 for the NACA 64A010A aiffoil (at Computational Condition 1). Both sets of results were computed using a pulse amplitude of $0.1^{\circ}$. The inviscid and viscous pulse results show the same trends with respect to reduced frequency. Differences between the inviscid and viscous $c_{\ell_{\alpha}}$ results are largest for low values of $k$. "In the low $k$ range, viscosity decreased the magnitude of both the real and imaginary parts. At high values of reduced frequency, the two sets of results are nearly the same.

The effects of pulse amplitude on transonic unsteady airloads were investigated by obtaining unsteady forces for successively increased pulse amplitudes. Calculations were performed using both inviscid and viscous pulse analyses. The inviscid pulse amplitude computations (not shown here) indicate that the unsteady forces are relatively independent of amplitude. Viscous pulse amplitude computations showing effects of amplitude for $c_{2}$ are presented in Fig. 6 for the NACA 64AOIOA airfoil (at Computational Condition 1). Comparison between viscous pulse computations at $\alpha_{0}=0.1^{\circ}$ with the computations at $\alpha_{0}=1.0^{\circ}$ shows some differences over the range of $k$ plotted. With increased pulse amplitude, the magnitudes of the real and imaginary parts of $c_{\ell_{\alpha}}$ are increased.

Unsteady experimental data from Davis, 16 and the computational results of Houwink 20 and Guruswamy and Goorjian ${ }^{4}$ are also plotted in Fig. 6 for further comparison. The experimental data of Ref. 16 was obtained using a harmonic pitch amplitude of $\alpha_{0} \approx 1.0^{\circ}$. The unsteady 


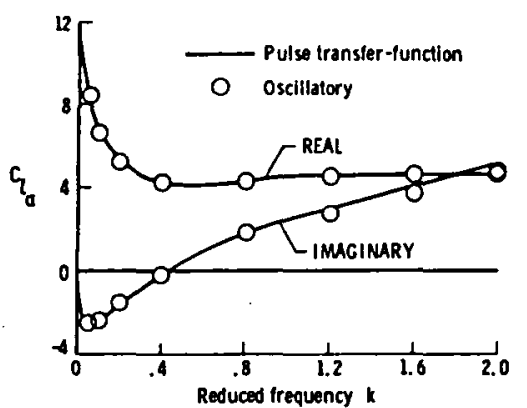

Fig. 4 Lift coefficient due to pitch about the quarter chord, $C_{l_{\alpha}}$, calculated using pulse and oscillatory analyses with viscous effects, for the NACA 64A010A airfoll at $M=0.796, \alpha_{m}=-0.21^{\circ}$, and $\alpha_{0}=0.1^{\circ}$.

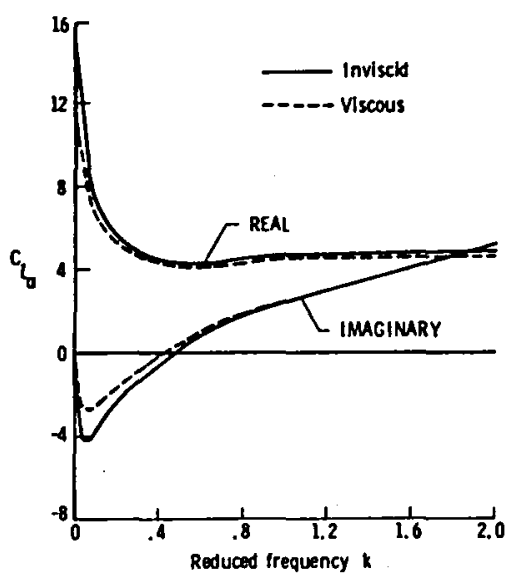

Fig. 5 Comparison of inviscid and viscous pulse results for the lift coefficient due to pitch about the quarter chord, $c_{\ell_{\alpha}}$, for the NACA $64 A 010 \mathrm{~A}$ airfoil at $M=0.796, \alpha_{m}=-0.21^{\circ}$, and $\alpha_{0}=0.1^{\circ}$.

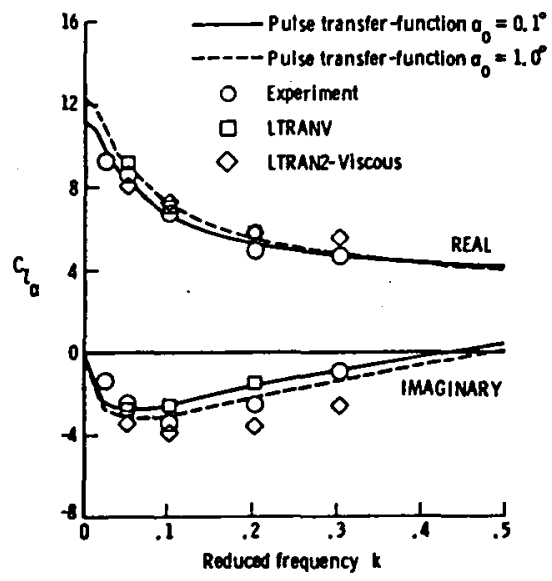

Fig. 6 Comparison of pulse results with experimental data, LTRANV and LTRAN2-Viscous results for the lift coefficient due to pitch about the quarter chord, $c_{\ell}$, for the NACA $64 A 010 A$ afrfoil at $M=0.796$ and $\alpha_{m}=-0.21^{\circ}$. forces of Ref. 20 were calculated with the LTRANV code using $\alpha_{0}=1.0^{\circ}, M=0.8$, and $\alpha_{m}=0^{\circ}$. The unsteady forces of Ref, 4 were calculated with the viscous version of LTRAN2 at Computational Condition 1 using harmonic pitch amplitudes selected to match the experiment. As shown in Fig. 6 , the viscous pulse computations performed using $\alpha_{0}=0.1^{\circ}$ show good overall agreement with the experimental forces except for the imaginary values of $c_{\ell}$ at $k=0.1$ and $k=0.2$. Comparison of the $\alpha$ viscous pulse computations with the LTRANV airloads shows good overall agreement. The imaginary part of the LTRANV results compares well with the imaginary part of the viscous XTRAN2L results for pulse amplitude $\alpha_{0}=0.1^{\circ}$, whereas the real part of the LTRANV results are in better agreement with the real part of the viscous XTRAN2L results for pulse amplitude $\alpha_{0}=1.0^{\circ}$. Comparison of the viscous LTRAN2 results for $c_{l}$ with all of the other unsteady forces presefted in Fig. 6 shows good general agreement in the real part although the imaginary part is consistently overpredicted.

Comparisons between inviscid and viscous pulse results for the unsteady aerodynamic coefficients due to control surface motion, $c_{\ell}, c_{m_{\delta}}$, and $c_{h}$, are presented in Figs. $7(a), 7(b)$, and $f(c)$, respectively. These results were obtained for the NACA 64A006 airfoil with a trailing edge control surface of $25 \%$ chord (at Computational Condition 4). The inviscid and viscous pulse results were computed using pulse amplitude $\delta_{0}=0.1^{\circ}$. The differences between inviscid and viscous results for $c_{\ell_{\delta}}$ and $c_{m_{\delta}}$ are generally largest for low values of $k$. In the low $k$ range, the inclusion of viscosity decreased the magnitude of both the real and imaginary parts of $c_{l}$ and $c_{m s}$. At higher reduced frequency, the two sets of results are nearly the same. In the pulse results for $c_{h}$ shown in Fig. $7(\mathrm{c})$, viscosity decreased the value of both the real and imaginary parts throughout the range of $k$ plotted. Effects of pulse amplitude on $c_{l_{\delta}}$ and $c_{m_{\delta}}$ are similar to those shown in ${ }^{\ell}$ Fig. 6 . ${ }^{C_{\text {No }}}$ amplitude effect was noted for $\mathrm{Ch}_{\delta}$.

Unsteady experimental data of $2 w^{2} a^{18}$ and the computational results of Houwink ${ }^{20}$ (calculated using the LTRANV code) are also presented for further comparison (the harmonic control surface amplitude used in Refs. 18 and 20 was $\left.\delta_{0}=1.0^{\circ}\right)$. The viscous XTRAN2L pulse solution is in better agreement with experiment than the inviscid solution. Viscous pulse computations show good agreement with LTRANV results for the real and imaginary components of $c_{\ell_{\delta}}$ and the imaginary component of $c_{h_{\delta}}$

\section{Time-Marching Response Results}

Time-marching calculations were first performed using a small initial plunge displacement of $h(0)=0.001$ to determine the value of $Q$ which results in neutrally stable aeroelastic transients. The effects of amplitude on flutter were then investigated by obtaining timemarching responses for successively increased initial plunge displacement $h(0)$. In these calculations, the dynamic pressure $Q$ was set equal 


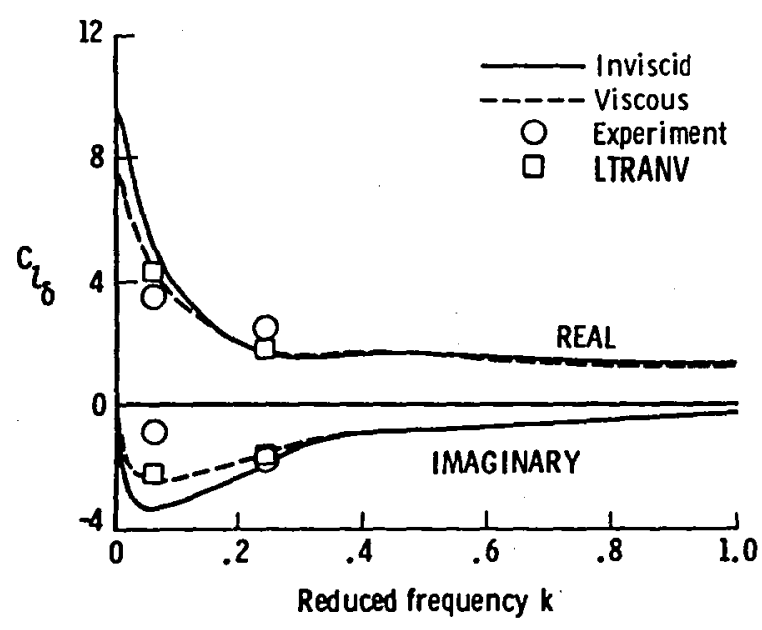

(a) lift coefficient due to control surface motion.

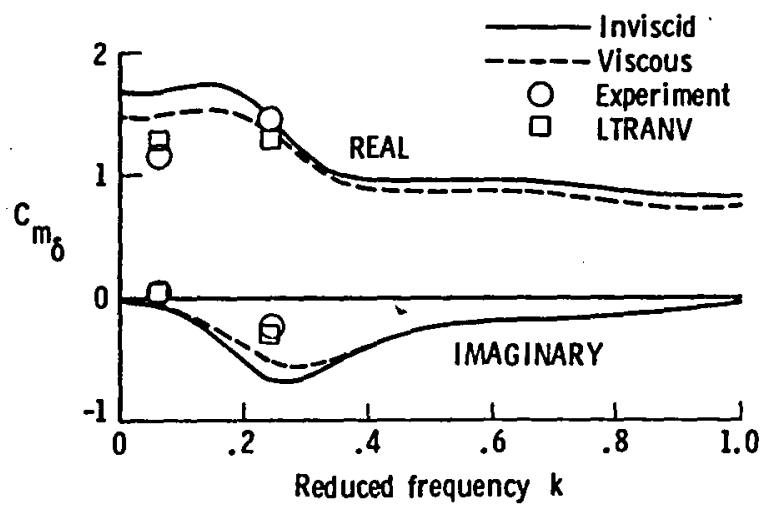

(b) moment coefficient due to control surface motion.

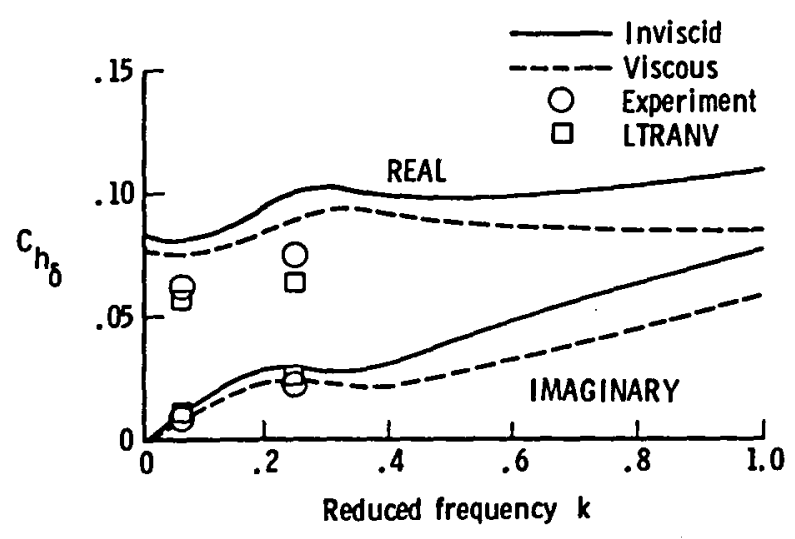

(c) hinge moment coefficient due to control surface motion.

Fig. 7 Comparison of control surface pulse results with experimental data and LTRANV results for the NACA 64A006 airfoil at $M=0.85, a_{m}=0^{\circ}$, and $\delta_{m}=0^{\circ}$; to the flutter dynamic pressure $Q_{F}$ previously determined using $h(0)=0.001$. Only representative responses for the NACA 64AO10A airfoil (at Computational Condition 1) are shown in this section for the : two example sets of structural parameter values listed in Table 2.

Example 1. - Neutrally stable plunge and pitch time-marching response histories are presented in Fig. 8. The responses have been normalized by the initial plunge displacement $h(0)=0.001$. Inviscid and viscous flutter dynamic pressure values used to calculate the aeroelastic transients are $Q_{F}=0.60$ and $Q_{F}=0.97$, respectively. The responses are bending dominated and are of constant amplitude after the higher frequency torsion mode transients, visible in the first 2-3 cycles of motion, have damped out. As shown in Fig. 8, the viscous responses have the same characteristics as the inviscid responses including nearly identical amplitudes. Accurate modal curve-fits of the viscous response histories were obtained using the method of Ref. 14. Damping and frequency estimates of the aeroelastic transients are plotted in the complex s-plane and are discussed in the following section.

The effects of amplitude on the inviscid and viscous time-marching flutter solutions of Fig. 8 are shown in Fig. 9. Plunge and pitch degrees-of-freedom exhibit similar characteristics and hence only the pitch responses are shown. Furthermore, the pitch responses have been normalized by $h(0)$ to allow for direct comparison between amplitude results. The pitch results for $h(0)=0.001$ in Fig. 9 are identical to the pitch resuits of Fig. 8. As shown in the top part of Fig. 9, the inviscid pitch responses become slightly divergent when the initial plunge displacement is increased by a factor of 100. The amplitude effect is consistent with similar results reported in Ref. 9, where increased amplitude had a small destabilizing effect on the flutter responses of the NACA $64 A 010 A$ and MBB-A3 airfolis for the structural parameter values of Example 1. As shown in the bottom part of Fig. 9, the viscous pitch responses show a similar but larger diverging trend for $h(0)=0.05$. At $h(0)=0.1$, viscous responses diverged rapidly and led to program failure. The inviscid pitch responses of Fig. 9 show a weak dependence on amplitude while the responses for the viscous case show a strong amplitude dependence.

Example 2. - Neutrally stable time-response histories for the structural parameter values of Example 2 are presented in Fig. 10. These responses are of constant amplitude and oscillate about an exponentially decaying mean. In contrast with Example 1, the inviscid and viscous flutter dynamic pressures are very close, $Q_{F}=0.88$ and $Q_{F}=0.86$, respectiveiy. The inviscid and viscous plunge response histories have approximately the same amplitude and frequency although the viscous response oscillates about a slightly different mean than the inviscid response. Similar trends are visible in the pitch responses of Fig. 10 (which are of smaller amplitude in comparison with the pitch responses of Example 1 (Fig. 8)). In contrast with the responses of Example 1, the higher frequency transient is not present in the 


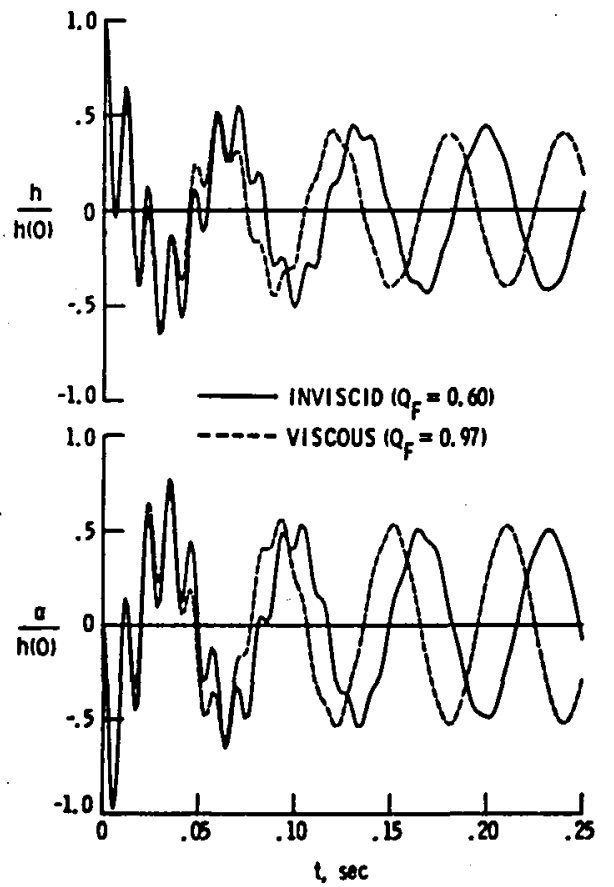

Fig. 8 NACA 64A010A neutrally stable time-marching response histories of Example 1 at $M=0.796$ and $\alpha_{m}=-0.21^{\circ}$.
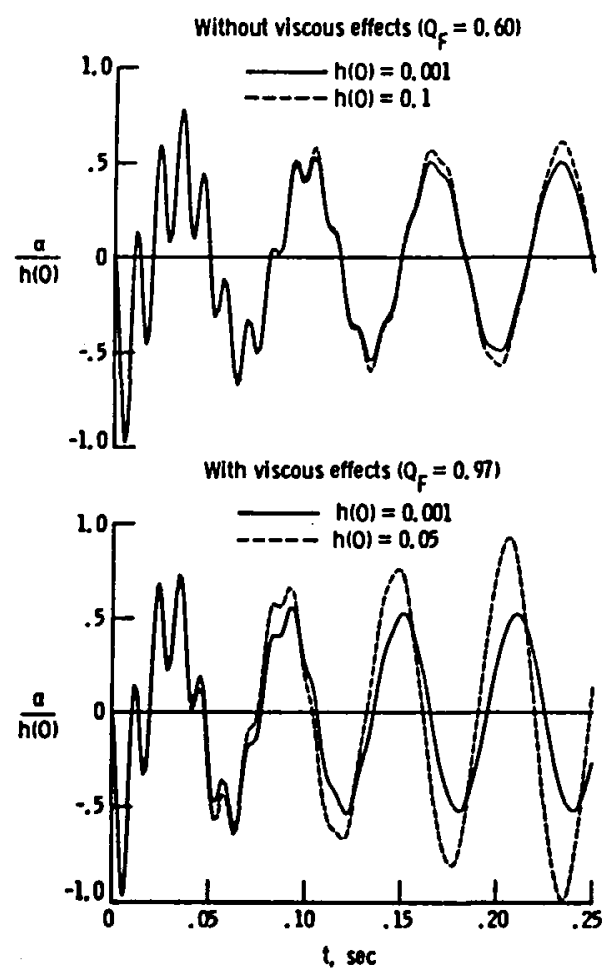

Fig. 9 Effects of amplitude on NACA 64A010A time-marching flutter solutions of Example 1 at $M=0.796$ and $a_{m}=-0.21^{\circ}$.

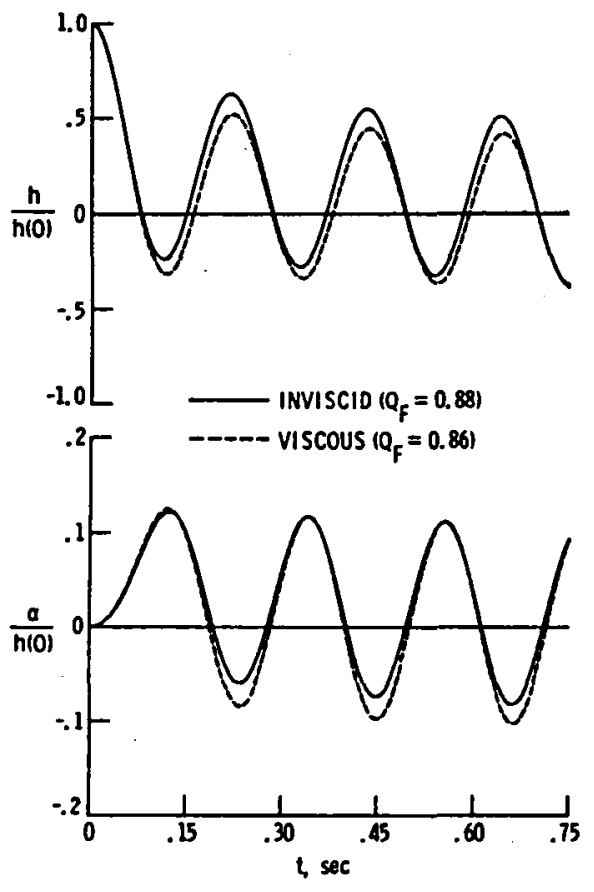

Fig. 10 NACA 64A010A neutrally stable time-marching response histories of Example 2 at $M=0.796$ and $\alpha_{m}=-0.21^{\circ}$.
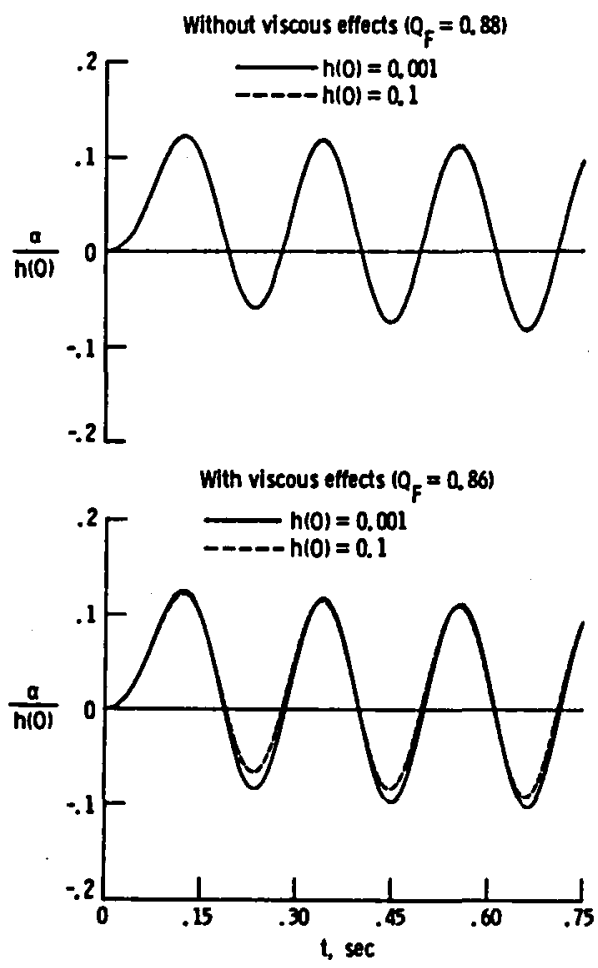

Fig. 11 Effects of amplitude on NACA 64A010A time-marching flutter solutions of Example 2 at $M=0.796$ and $\alpha_{m}=-0.21^{\circ}$. 
responses of Example 2. The higher frequency mode is highly damped and thus has a negligible contribution to the total response.

The effects of amplitude on the NACA 64A010A time-marching flutter solutions (at Computational Condition 1) are shown in Fig. 11. Inviscid pitch time-responses which are neutrally stable at $h(0)=0.001$ remain neutrally stable at $h(0)=0.1$. Viscous pitch responses are also neutrally stable when the initial plunge displacement is increased by a factor of 100. In contrast with the Example 1 responses of $\mathrm{Fig}$. 9, the Example 2 responses of Fig. 11 indicate that the flutter solution is relatively independent of amplitude.

\section{Pade Model Stability Results}

Pade model stability calculations were performed using transonic unsteady airloads determined by both inviscid and viscous pulse analyses. Pulse amplitudes were $h_{0}=0.001$ and $\alpha_{0}=0.1^{\circ}$. Results for the NACA 64A010A and $M B B-A 3$ airfoils are presented in this section for the two example sets of aeroelastic parameter values listed in Table 2. Comparisons between time-marching and Pade model flutter solutions for Example 1 and Example 2 are presented in Tables 3 and 4, respectively.

Example 1. - Pade model dynamic pressure root-Toci for the NACA 64A010A airfoil (at Computational Condition 1) are shown in Fig. 12(a). Inviscid and viscous root-loci are plotted. With increasing dynamic pressure the torsion dominated mode moves directly left in the stable left-half plane while the bending dominated root becomes the flutter mode. The inclusion of viscous effects increased damping in the bending mode and slightly lowered the torsion mode frequency. Time-marching damping and frequency estimates are plotted in Fig. 12 (a) for $Q=0.4,0.8$, and 1.2. As shown in the figure, the Pade model root-loci are in good agreement with the time-marching modal estimates in both the inviscid and viscous cases. The value of dynamic pressure at flutter is given by the $\sigma=0$ crossing. The Pade model flutter dynamic pressure values are $Q_{F}=0.62$ and $Q_{F}=1.00$ for the inviscid and viscous calculations, respectively.

Thus, viscous effects increased $Q_{F}$ by approximately $61 \%$. The Pade model QF values are within $3 \%$ of the small amplitude time-marching values presented in the previous section.

Pade model dynamic pressure root-loci for the MBB-A3 airfoil at Computational Condition 2 are shown in Fig. 12(b). Both inviscid and viscous root-loci for the bending and torsion modes are plotted. Time-marching modal estimates are also plotted in Fig. 12(b) for $Q=0.2,0.4,0.6$, and 0.8 . Pade model results are in good overall agreement with time-marching damping and frequency values. Pade model dynamic pressures at flutter are $Q_{F}=0.25$ and $Q_{F}=0.55$ for the inviscid and viscous MBB-A3 calculations, respectively. The inclusion of viscous effects increased the flutter dynamic pressure by approximately $124 \%$ in comparison with the inviscid calculation. Pade model $Q_{F}$ values differ from the small amplitude timemarching $Q_{F}$ values listed in Table 3 by $7 \%$ and $22 \%$ for the inviscid and viscous cases, respectively. These differences may be attributed to the pulse amplitude effects on viscous transonic unsteady airloads similar to that shown in Fig. 6 and to the amplitude dependence of the flutter responses of Example 1 shown for the NACA 64A010A airfoil in Fig. 9. The larger differences between Pade model and time-marching $Q_{F}$ values in the viscous case may also be attributed to the more computationally sensitive nature of the viscous XTRAN2L calculations in contrast with the relatively routine inviscid calculations.

The MBB-A3 root-loci of Fig. $12(\mathrm{~b})$ are very similar to the NACA 64AO10A root-loci of Fig. 12(a). The inviscid flutter characteristics of these two airfoils were shown by Bland and Edwards ${ }^{10}$ to be nearly identical when the steady shock strengths and locations were matched. The large differences in $Q_{F}$ between the two airfoils presented here may therefore be attributed to differences in steady shock strength and location as shown by comparison of Figs. 1 and 2. Also, since the shock on the MBB-A3 airfoil (Computational Condition 2, Fig. 2) is stronger in comparison with the shock on the NACA 64AO10A airfoll (Fig. 1), viscosity has more influence

Table 3 Comparisons between inviscid and viscous time-marching and Pade model flutter solutions for Example 1 .

\begin{tabular}{|c|c|c|c|c|c|c|c|c|}
\hline \multirow{3}{*}{$\begin{array}{l}\text { Airfoils and } \\
\text { Computational Conditions }\end{array}$} & \multicolumn{4}{|c|}{ INV ISCID } & \multicolumn{4}{|c|}{ VISCOUS } \\
\hline & \multicolumn{2}{|c|}{ Time-marching } & \multicolumn{2}{|c|}{ Pade Model } & \multicolumn{2}{|c|}{ Time-marching } & \multicolumn{2}{|c|}{ Pade Model } \\
\hline & $\overline{Q_{F}}$ & $\omega_{F} / \omega_{\alpha}$ & $\nabla_{F}$ & $\omega_{F} / \omega_{\alpha}$ & $\overline{Q_{F}}$ & $\omega_{F} / \omega_{\alpha}$ & $Q_{F}$ & $\omega_{F} / \omega_{\alpha}$ \\
\hline $\begin{array}{l}\text { NACA } 64 A 010 A \\
M=0.796, \alpha_{m}=-0.21^{\circ} \\
\operatorname{Re}=12.56 \times 10^{6}\end{array}$ & 0.60 & 0.96 & 0.62 & 0.96 & 0.97 & 1.06 & 1.00 & 1.06 \\
\hline $\begin{array}{l}M B B-A 3 \\
M=0.765, \alpha_{m_{6}}=1.5^{\circ} \\
\operatorname{Re}=6.0 \times 10^{6}\end{array}$ & 0.27 & 0.82 & 0.25 & 0.81 & 0.46 & 0.89 & 0.55 & 0.92 \\
\hline $\begin{array}{l}\text { MBB-A3 } \\
M=0.7557, \alpha_{m}=1.3^{\circ}\end{array}$ & 0.65 & 0.97 & 0.74 & 1.00 & & & & \\
\hline
\end{tabular}



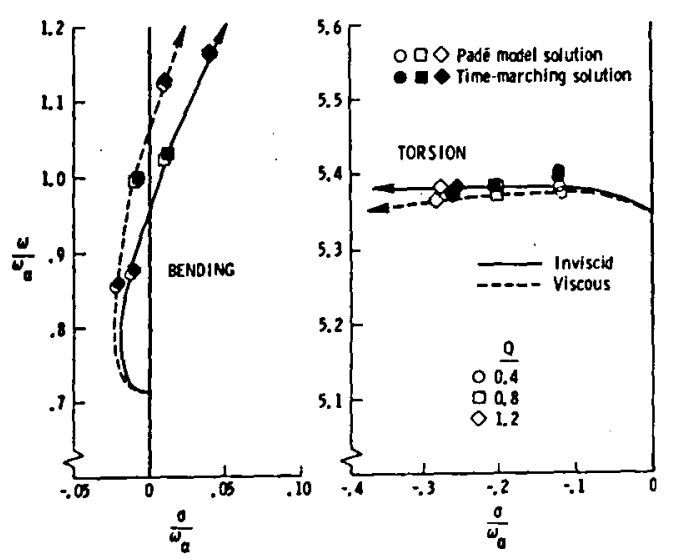

(a) NACA 64A010A airfoil at

$M=0.796$ and $\alpha_{m}=-0.21^{\circ}$.
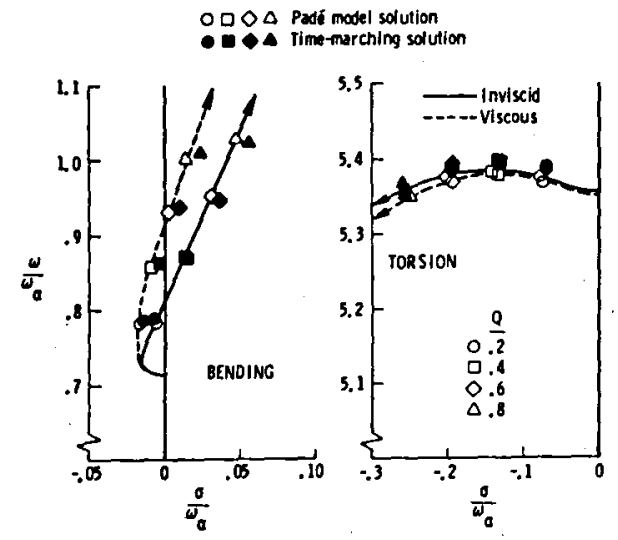

(b) $M B B-A 3$ alrfoil at $M=0.765$ and $\alpha_{m}=1.5^{\circ}$.
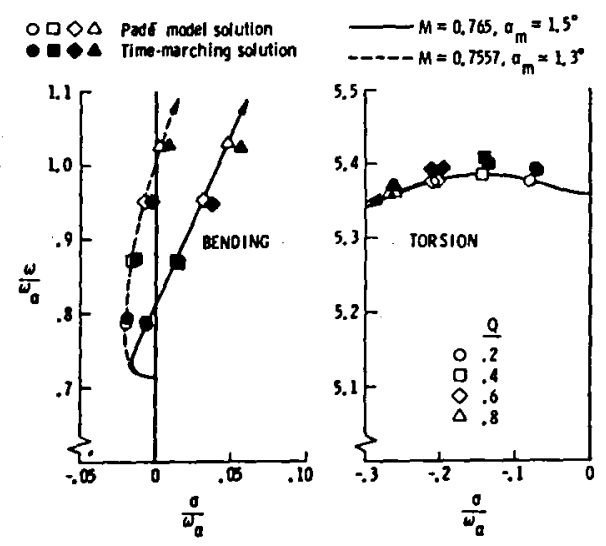

(c) $M B B-A 3$ airfoll at $M=0.765$, $\alpha_{m}=1.5^{\circ}$ and $M=0.7557$, $\alpha_{m}=1.3^{\circ}$.

Fig. 12 Effects of viscosity on Pade model and time-marching dynamic pressure rootloci of Example 1;

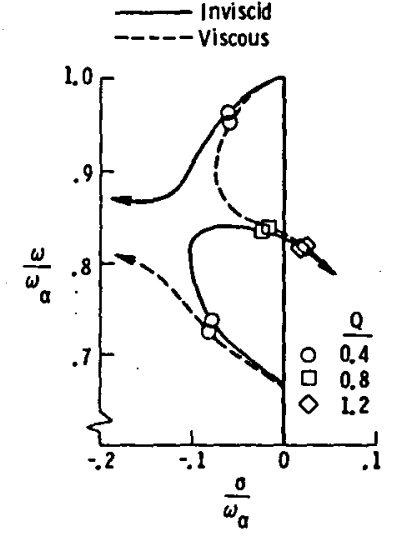

(a) NACA 64A010A airfoil at $M=0.796$ and $\alpha_{m}=-0.21^{\circ}$.

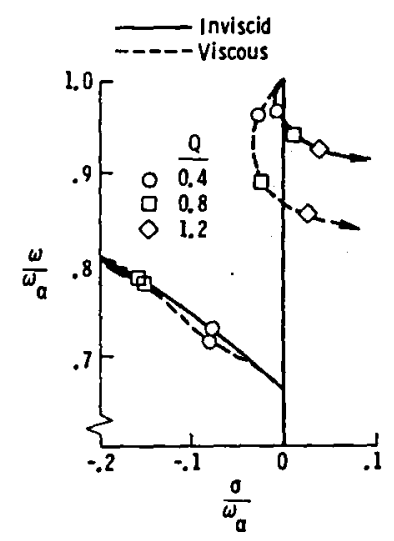

(b) $M B B-A 3$ airfoil at $M=0.765$ and $\alpha_{\mathrm{m}}=1.5^{\circ}$.
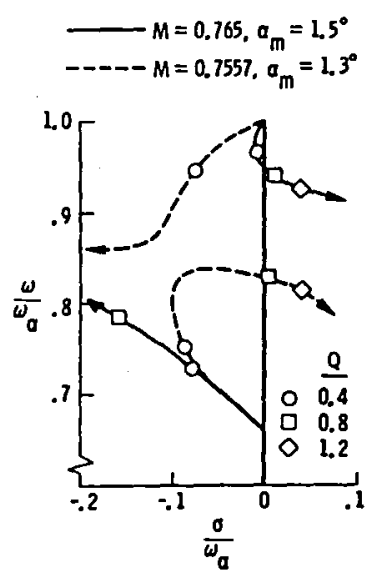

(c) $M B B-A 3$ airfoil at $M=0.765$, $\alpha_{m}=1.5^{\circ}$ and $M=0.7557$, $\alpha_{m}=1.3^{\circ}$.

Fig. 13 Effects of viscosity on Pade model dynamic pressure root-loci of Example 2; 
Table 4 Comparisons between inviscid and viscous time-marching and Pade model flutter solutions for Example 2.

\begin{tabular}{|c|c|c|c|c|c|c|c|c|}
\hline \multirow{3}{*}{$\begin{array}{l}\text { Airfoils and } \\
\text { Computational Conditions }\end{array}$} & \multicolumn{4}{|c|}{ INVISCIO } & \multicolumn{4}{|c|}{ VISCOUS } \\
\hline & \multicolumn{2}{|c|}{ Time-marching } & \multicolumn{2}{|c|}{ Pade Model } & \multicolumn{2}{|c|}{ Time-marching } & \multicolumn{2}{|c|}{ Pade Model } \\
\hline & $Q_{F}$ & $\omega_{F} / \omega_{\alpha}$ & $Q_{F}$ & $\omega_{F} / \omega_{\alpha}$ & $Q_{F}$ & $\omega_{F} / \omega_{\alpha}$ & $Q_{F}$ & $\omega_{F} / \omega_{\alpha}$ \\
\hline $\begin{array}{l}\text { NACA } 64 A 010 \mathrm{~A} \\
M=0.796, \alpha_{\mathrm{m}}=-0.21^{\circ} \\
\operatorname{Re}=12.56 \times 10^{6}\end{array}$ & 0.88 & 0.84 & 0.97 & 0.83 & 0.86 & 0.84 & 0.92 & 0.83 \\
\hline $\begin{array}{l}M B B-A 3 \\
M=0.765, \alpha_{m_{b}}=1.5^{\circ} \\
\operatorname{Re}=6.0 \times 10^{6}\end{array}$ & 0.61 & 0.94 & 0.58 & 0.95 & 0.86 & 0.89 & 0.96 & 0.80 \\
\hline $\begin{array}{l}\text { MBB-A3 } \\
M=0.7557, \alpha_{m}=1.3^{\circ}\end{array}$ & 0.76 & 0.83 & 0.76 & 0.83 & & & & \\
\hline
\end{tabular}

on the steady pressures and hence larger changes in $Q_{F}$ result.

Inviscid dynamic pressure root-loci for the MBB-A3 airfoil at Computational Conditions 2 and 3 are shown in Fig. 12(c). The Pade model flutter dynamic pressure values are $Q_{F}=0.25$ at Computational Condition 2 and $Q_{F}=0.74$ at Computational Condition 3. The small decreases in Mach number and mean angle of attack in the inviscid calculations result in similar changes in the aeroelastic root-loci (Fig. $12(\mathrm{c})$ ) as occurred with the inclusion of viscous effects at Computational Condition 2 (Fig. 12(b)). The three sets of computations performed for the MBB-A3 airfoil resulted in steady shock locations of $63 \%, 61 \%$, and $51 \%$ chord and monotonically decreased shock strength as shown in the steady pressure distributions of Fig. 2. The corresponding Pade model flutter dynamic pressure values are $Q_{F}=0.25,0.55$, and 0.74 , respectively. The corresponding time-marching flutter dynamic pressure values are $Q_{F}=0.27$, 0.46 , and 0.65 , respectively. With successively decreased shock strength and forward shock displacement, there is a monotonic increase in $Q_{F}$. Pade model $Q_{F}$ values for the MBB-A3 airfoil were typically nonconservative for Example 1 which may be attributed to the amplitude effects discussed earlier.

Example 2. - Inviscid and viscous Pade model dynamic pressure root-loci for the NACA 64A010A airfoil (at Computational Condition 1) are shown in Fig. 13(a). In general, the inclusion of viscous effects increased damping in the lower frequency mode and decreased damping in the higher frequency mode. A switch in modal origin of the flutter mode occurs for this case with the addition of viscosity. The viscous computations give a fiutter dynamic pressure value of $Q_{F}=0.92$ which is slightly less than the value of $Q_{F}=0.97$ given by the inviscid computations. The inviscid Pade model flutter dynamic pressure is within $10 \%$ of the timemarching flutter dynamic pressure, $Q_{F}=0.88$. Viscous Pade model and time-marching flutter dynamic pressures differ by only $7 \%$, as listed in Table 4. The Pade model root-locus results were in qualitative agreement with the timemarching results. Damping and frequency estimates from the time-marching transients were not obtained, though, because of the closeness in frequency of the two aeroelastic modes.

Pade model dynamic pressure root-loci for the MBB-A3 airfoil at Computational Condition 2 are shown in Fig. 13(b). The inclusion of viscous effects increased damping and decreased frequency in the higher frequency mode. The lower frequency mode shows only small changes as a result of including viscosity. Dynamic pressures at flutter are $Q_{F}=0.58$ and $Q_{F}=0.96$ for the inviscid and viscous cases, respectively. Pade model $Q_{F}$ values differ from the small amplitude time-marching $Q_{F}$ values listed in Table 4 by $5 \%$ and $12 \%$ for the inviscid and viscous calculations, respectively. The inclusion of viscous effects resulted in a $66 \%$ increase in flutter dynamic pressure, which is in contrast with the small changes in $\mathrm{QF}_{\mathrm{F}}$ found for the NACA 64A010A airfoil (Fig. 13(a)). The large increase in $Q_{F}$ between inviscid and viscous MBB-A3 cases at Computational Condition 2 is due to the stronger steady shock in comparison with that of the NACA 64AO1OA airfoil (at Computational Condition 1 ).

Inviscid Pade model dynamic pressure rootloci for the $M B B-A 3$ airfoll at Computational Conditions 2 and 3 are presented in Fig. 13(c). The flutter dynamic pressure at Computationa 1 Condition 3 is $Q_{F}=0.76$ which is the same as the time-marching $Q_{F}$ value listed in Table 4 . The small decreases in Mach number and mean angle of attack' in the inviscid calculations result in decreased damping in the lower frequency mode and increased damping in the higher frequency mode. A change in the modal origin of the flutter mode (Fig. 13(c)) also occurs which is opposite to that for the NACA 64A010A atrfoll of Example 2 with the inclusion of viscous effects (Fig. 13(a)). Pade model flutter dynamic pressure values for Example 2 were typically nonconservative which was similar to that found for Example 1.

\section{Concluding Remarks}

Viscous effects on transonic airfotl stabllity and response have been investigated 
based on the use of the XTRAN2L transonic smalldisturbance code. Aeroelastic results were presented for the NACA 64A010A and MBB-A3 airfoils. Aerodynamic calculations including viscous effects were performed using an integral boundary layer model coupled to the inviscid potential outer flow in a quasi-steady fashion.

Transonic unsteady aerodynamic coefficients required for stability calculations were computed using a pulse transfer-function analysis. Excellent agreement was found between the pulse transfer-function and oscillatory airloads thus demonstrating the ability of the pulse analysis to include viscous effects. Inviscid and viscous pulse results showed the same trends with respect to reduced frequency although differences occur for low values of $k$. Viscous pulse computations showed better overall agreement with experimental data than the inviscid pulse computations.

Nonlinear time-marching flutter solutions were obtained which showed the effects of viscosity and amplitude on airfoil response behavior and flutter. A two degree-of-freedom (plunge and pitch) deroelastic system was considered. Representative responses for the NACA 64A010A airfoil were presented for two different example sets of aeroelastic parameter values. In both examples, the viscous responses at flutter had the same characteristics as the inviscid responses including very similar amplitudes. In the first example, which had bending and torsion modes similar to those of a streamwise section near the tip of a sweptback wing, large increases in flutter dynamic pressure $Q_{F}$ resulted with the inclusion of viscous effects. Also, the inviscid flutter responses showed a weak dependence on amplitude while the viscous flutter responses showed a strong amplitude dependence. In the second example, which had bending and torsion frequencies that were close together, viscosity had little effect on flutter dynamic pressure. Also in the second example, the time-marching flutter responses were relatively independent of amplitude.

Aeroelastic stability analyses were performed using a Pade state-space aeroelastic model. Dynamic pressure root-1oci were presented for the NACA 64A010A and MBB-A3 airfolls for the two example sets of aeroelastic parameter values. Pade model root-loci for the first example considered, showed that viscous effects lowered the torsion mode frequency and increased damping in the bending mode thus delaying the onset of flutter. Changes in flutter dynamic pressure were correlated with changes in steady shock strength and location. Inclusion of viscous effects weakened the shock on the MBB-A3 airfoil more than that of the NACA 64A010A airfoil and consequently caused larger increases in $Q_{F}$. For the three sets of computations performed for the MBB-A3 airfoil, decreased shock strength and forward shock displacement produced increased $Q_{F}$ values. In general, the Pade model root-1oci were in good overall agreement with time-marching damping and frequency estimates in both the inviscid and viscous cases. In the second example, viscosity caused an insignificant change in the NACA 64A010A flutter dynamic pressure. For the
MBB-A3 airfoil, a $66 \%$ increase in $Q_{F}$ resulted with the inclusion of viscous effects which was attributed to a stronger steady shock in comparison with the NACA 64A010A airfoil. Pade model flutter dynamic pressure values for both example sets of aeroelastic parameter values were typically nonconservative. Differences between Pade model and time-marching values for $Q_{F}$ are attributed to pulse amplitude effects on viscous transonic unsteady airloads and to the amplitude dependence of the flutter responses in the first example considered.

\section{Acknowledgements}

This work constitutes a part of the first author's M.S. thesis and was supported by the NASA Langley Graduate Aeronautics Program under contract NAG-1-372.

\section{References}

${ }^{1}$ Ballhaus, W. F., "Computational Aerodynamics and Supercomputers," NASA TM 85887 , January, 1984.

${ }^{2}$ Ballhaus, W. F., and Goorjian, P. M., "Implicit Finite-Difference Computations of Unsteady Transonic Flow about Airfoils," AIAA Journal, Vol. 15, No. 12, December, 1977.

${ }^{3}$ Rizzetta, D. P., "Procedures for the Computation of Unsteady Transonic Flows Including Viscous Effects," NASA CR 166249, January, 1982.

${ }^{4}$ Guruswamy, P. and Goorjian, P. M., "Effects of Viscosity on Transonic-Aerodynamic and Aeroelastic Characteristics of Oscillating Airfolls," Journal of Aircraft, Vol. 21, September, 1984, pp. 700-707.

${ }^{5}$ Houwink, R., "Results of a New Version of the LTRAN2-NLR Code (LTRANV) for Unsteady Viscous Transonic Flow Computations," NLR TR 81078U, National Aerospace Laboratory, the Netheriands, July, 1981.

${ }^{6}$ Houwink, R. and van der Vooren, J., "Improved Version of LTRAN2 for Unsteady Transonic Flow Computations," AIAA Journal, Vol. 18, No. 8, August, 1980 , pp. 1008-1010.

7Howlett, J. T., "Efficient Self-Consistent Viscous-Inviscid Solutions for Unsteady Transonic Flow," AIAA Paper No. 85-0482, presented at the AIAA 23rd Aerospace Sciences Meeting and Technical Display, Reno, NV, January 14-17, 1985.

${ }^{8}$ Whit low, W., Jr., "XTRAN2L: A Program for Solving the General Frequency Unsteady Transonic Small Disturbance Equation," NASA TM 85723, November, 1983.

${ }^{9}$ Edwards, J. W., Bennett, R. M., Whitlow, W., Jr., and Seidel, D. A., "Time Marching Transonic Flutter Solutions Including Angle of Attack Effects, "Proceedings of the AIAA/ASME/ ASCE/AHS 23rd Structures, Structural Dynamics and Materials Conference, New Orleans, LA, May 10-12, 1982, pp. 220-233. 
${ }^{10}$ Bland, S. R., and Edwards, J. W., "Airfoil Shape and Thickness Effects on Transonic Airloads and Flutter," Journal of Aircraft, Vol. 21, March, 1984, pp. 209-217.

''Batina, J. T., and Yang, T. Y., "Application of Transonic Codes to Aeroelastic Modeling of Airfoils Including Active Controls," Journal of Aircraft, Vol. 21, August, 1984, pp. 623-630.

${ }^{12}$ Batina, J. T., and Yang, T. Y., "Transonic Calculation of Airfoil Stability and Response with Active Controls," AIAA Paper No. 84-0873, presented at the AIAA/ASME/ASCE/AHS 25th Structures, Structural Dynamics, and Materials Conference, Palm Springs, CA, May 14-16, 1984, also NASA TM 85770, March, 1984.

${ }^{13}$ Seidel, D. A., Bennett, R. M., and Whitlow, W., Jr., "An Exploratory Study of Finite-Difference Grids for Transonic Unsteady Aerodynamics," AIAA Paper No. 83-0503, presented at the AIAA 21st Aerospace Sciences Meeting and Technical Display, Reno, NV, January 10-13, 1983, also NASA TM 84583, December, 1982.

${ }^{14}$ Bennett, R. M., and Desmarais, R. N., "Curve Fitting of Aeroelastic Transient Response Data with Exponential Functions in Flutter Testing Techniques," NASA SP-415, 1975, pp. 43-58.
15Bland, S. R., "AGARD Two-Dimensional Aeroelastic Configurations," AGARD-AR-156, August, 1979.

${ }^{16}$ Davis, S. S., "NACA 64A010 (NASA Ames Model) Oscillatory Pitching" in "Compendium of Unsteady Aerodynamic Measurements," AGARD Report No. 702, August, 1982.

17Bucciantini, G., Oggiano, M. S., and Onorato, M., "Supercritical Airfoil MBB-A3, Surface Pressure Distributions, Wake and Boundary Condition Measurements," AGARD-AR-138, May, 1979, pp. A8-1-AB-25.

${ }^{18}$ Zwaan, R. J., "NACA 64A006 Oscillating Flap" in "Compendium of Unsteady Aerodynamic Measurements," AGARD Report No. 702, August, 1982.

${ }^{19}$ Isogai, K., "Numerical Study of Transonic Flutter of a Two-Dimensional Airfoil," NAL TR-617T, National Aerospace Laboratory, Tokyo, Japan, JuTy, 1980.

${ }^{20}$ Houwink, R., "Unsteady Transonic Flow Computations for AGARD 2-D Aeroelastic Configurations," NLR Merno AE-83-004 U, National Aerospace Laboratory, the Netherlands, March, 1983. 


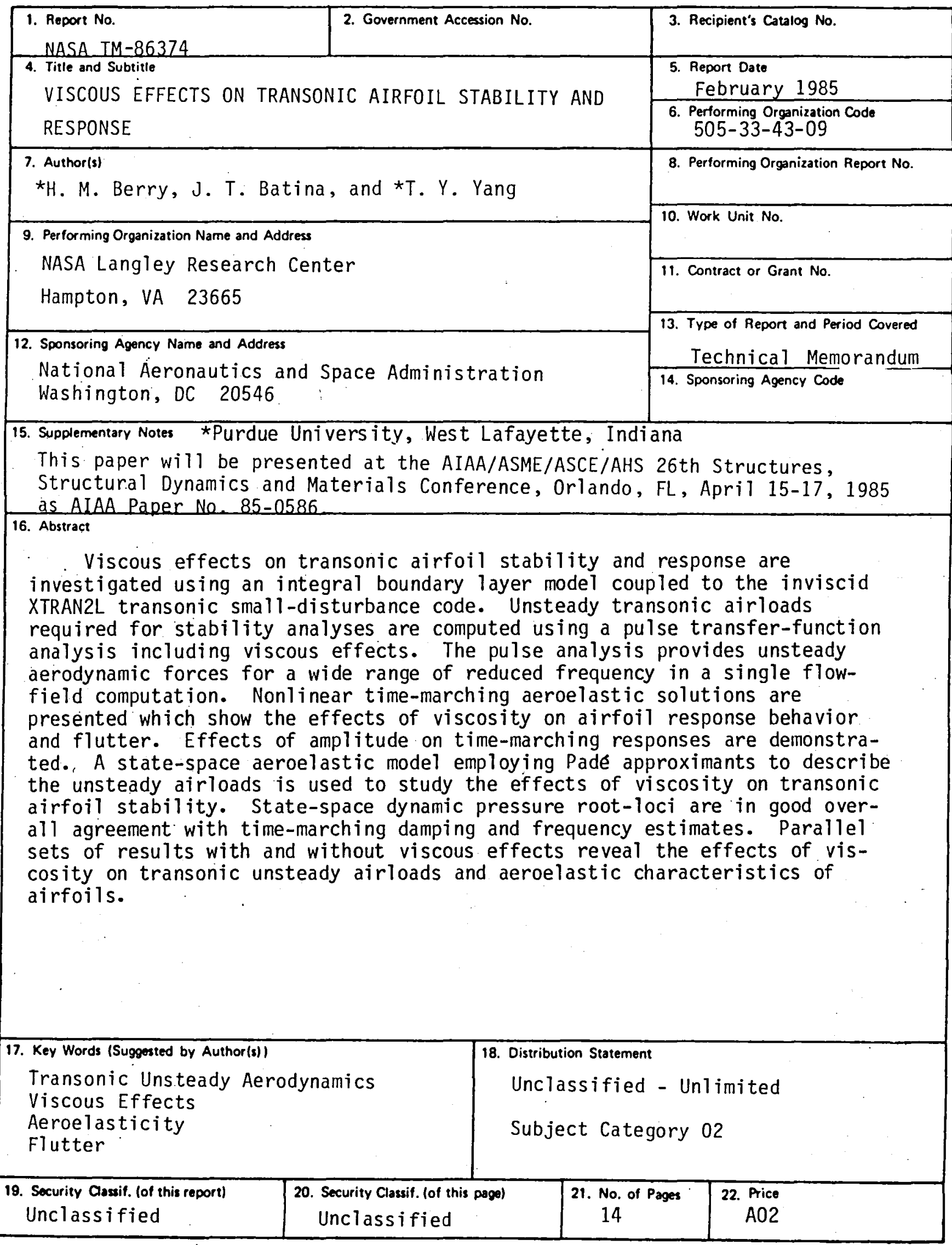


End of Document 